\title{
On induced permutation matrices and the symmetric group
}

By A. C. Aitken, University of Edinburgh.

(Received 18th November, 1935. Read 6th December, 1935.)

1. The Matrix Representation OF PERMUtations.

The $n$ ! operations $\mathrm{A}_{i}$ of permutations upon $n$ different ordered symbols correspond to $n !$ matrices $A_{i}$ of the $n^{\text {th }}$ order, which have in each row and in each column only one non-zero element, namely a unit. Such matrices $A_{i}$ are called permutation matrices, since their effect in premultiplying an arbitrary column vector $x=\left\{x_{1} x_{2} \ldots x_{n}\right\}$ is to impress the permutation $\mathrm{A}_{i}$ upon the elements $x_{i}$. For example the six matrices of the third order

$$
A_{0}=\left[\begin{array}{lll}
1 & \cdot & \cdot \\
\cdot & 1 & . \\
\cdot & \cdot & 1
\end{array}\right], \quad A_{1}=\left[\begin{array}{lll}
1 & \cdot & \cdot \\
\cdot & . & 1 \\
\cdot & 1 & .
\end{array}\right], \ldots, \quad A_{5}=\left[\begin{array}{ccc}
\cdot & \cdot & 1 \\
\cdot & 1 & \cdot \\
1 & \cdot & \cdot
\end{array}\right]
$$

are permutation matrices. It is convenient to denote them by

$$
A_{0}=[123], \quad A_{1}=[132], \ldots, A_{5}=[321],
$$

where the bracketed indices refer to the permutations of natural order. Clearly the relation $\mathrm{A}_{i} \mathrm{~A}_{j}=\mathrm{A}_{k}$ entails the matrix relation $A_{i} A_{j}=A_{k}$; in other words, the $n !$ matrices $A_{i}$ give a matrix representation of the symmetric group of order $n !$.

Permutations $\mathbf{A}_{i}$ may be classified in the usual manner according to the cycles which they contain. For example, denoting by $(\alpha \beta \gamma)$ the operation which replaces the order $a \beta \gamma$ by $\beta \gamma a$, we may write [2134] as [(12) 34], and [4321] as [(14)(23)]. Symbols unaltered by any operation count as cycles on one symbol.

The number of symbols affected by the respective cycles in $A_{i}$, when expressed as parts of a partible integer, as for example $2+1+1$ symbols in [(12) 34], or $2+2$ symbols in [(14)(23)], constitutes a

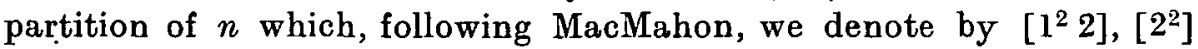
and the like. We choose the ascending order of parts in a partition in preference to the more usual descending order for reasons of convenience. 
It is well known (cf. Burnside, Theory of Groups, 2nd Edition, 1911, p. 7) that permutations of the same cycle-type, that is, typified by the same partition of $n$, are connected by the relation of similarity or equivalence

$$
\mathrm{A}_{i}=\mathrm{HA}_{j} \mathrm{H}^{-1} \text {. }
$$

Also, by mere inspection of any permutation matrix $A_{i}$, we note that cycles on one symbol correspond to unit elements in the diagonal, while cycles on more than one symbol correspond to chains of unit elements all off the diagonal. Hence the sum of the diagonal elements of $A_{i}$, the trace or spur or characteristic, is equal to the number of cycles on one symbol in the permutation $A_{i}$.

Finally it may be remarked that when $\mathbf{A}_{i}$ corresponds to a single cycle on $n$ symbols, the characteristic determinant $\left|A_{i}-x I\right|$ is equal to $1-x^{n}$. Hence, and more generally, since no two cycles in $A_{i}$ have an index in common, the submatrices corresponding to individual cycles in an $A_{i}$ of cycle-type or class

$$
\left[p_{1}^{a_{1}} p_{2}^{a_{2}} \ldots \ldots\right]
$$

are isolated, and the characteristic determinant of such an $A_{i}$ is therefore

$$
\left|A_{i}-x I\right|=\left(1-x^{p_{1}}\right)^{a_{1}}\left(1-x^{p_{2}}\right)^{\alpha_{2}} \ldots
$$

2. INDUCED MATRICES.

We consider any matrix $A$ (not necessarily a permutation matrix) of the $n^{\text {th }}$ order, and an arbitrary vector transformed by it:

$$
A\left\{x_{1} x_{2} \ldots x_{n}\right\}=\left\{y_{1} y_{2} \ldots y_{n}\right\} \text {. }
$$

The powers and products of the elements $x_{i}$, of degree $r$, may be arranged in dictionary order of suffixes as a vector $x^{[r]}$ of $\left(\begin{array}{c}n+r-1 \\ r\end{array}\right)$ elements; and the $r$-ary powers and products of the $y_{i}$ in the same way. The matrix which transforms the vector $x^{[r]}$ into $y^{[r]}$ is called the $r^{\text {th }}$ induced matrix ("power transformation") of $A$. It is denoted by $A^{[r]}$; and by convention $A^{[0]}$ is taken to be the scalar number 1 .

Induced matrices possess, as is well known, multiplicative properties, readily established from their definition; namely that if $A B=C$ then

while

$$
A^{[r]} B^{[r]}=C^{[r]}
$$

$$
\left(A^{[r]}\right)^{-1}=\left(A^{-1}\right)^{[r]}, \quad|A| \neq 0 .
$$


It follows at once that the induced matrices of permutation matrices, $A_{i}^{[r]}$, have the group properties of the $A_{i}$, and therefore constitute a matrix representation of the group $\mathrm{A}_{i}$. Hence the vector of $n$ ! elements given by their traces is a group character. For example when $n=3$ we have the induced characters as given below.

\begin{tabular}{r|ccc|} 
Class & {$\left[1^{3}\right]$} & {$[12]$} & {$[3]$} \\
No. in Class & 1 & 3 & 2 \\
\hline$A^{[0]}$ & 1 & 1 & 1 \\
$A^{[1]}$ & 3 & 1 &. \\
$A^{[2]}$ & 6 & 2 &. \\
$A^{[3]}$ & 10 & 2 & 1 \\
$\ldots \ldots$ & $\ldots \ldots$ & $\ldots$
\end{tabular}

With the exception of the unit or scalar character corresponding to $A^{[0]}$ these induced characters are not simple; the sum of the squares of their elements exceeds $n$, the order of the group, and so the representations $A^{[1]}, A^{[2]}, \ldots$ are reducible. Further, the characters are not all linearly independent; for example the character of $A^{[2]}$ is merely the double of that of $A^{[1]}$.

It is proposed to investigate for the general case what these characters are, and more particularly what relation they bear to the table of simple group characters.

It is possible to write down these induced characters by rule, from first principles. For example, if $[\alpha \beta \gamma]$ is the umbral symbol for any matrix $A$ (not necessarily a permutation matrix) of the third order, the matrix $A^{[2]}$ may be umbrally described by paired symbols, thus,

$$
A^{[2]} \equiv[\alpha \alpha a \beta \alpha \gamma \beta \beta \beta \gamma \gamma \gamma]
$$

and in general $A^{[r]}$ by $r$-ary symbols, corresponding to $r$-ary powers and products transformed by $A^{[r]}$. In the case of a permutation matrix $A_{i} \equiv[\alpha \beta \gamma]$, any $r$-ary symbol unaltered in its constituent indices by the cycles in $A_{i}$ corresponds to a unit in the diagonal of $A_{i}^{[r]}$, while any $r$-ary symbol that is altered corresponds to a unit off the diagonal. Hence the trace of $A_{i}^{[r]}$, which is evidently a permutation matrix, is the number of $r$-ary symbols unaltered by $A_{i}$.

Consider for example $A^{[3]}$, where $n=4$. The cycle-types are

$[1234], \quad[12(34)], \quad[(12)(34)], \quad[1(234)], \quad[(1234)]$. 
Trace.

Under [1234] each of the 20 ternary symbols is unaltered. $\quad 20$

Under $[12(34)], 111,112,122,222,134,234$ remain unaltered. 6 Under [1(234)] only 111,234 remain unaltered. 2 All other cycles alter every ternary symbol. 0

Hence the (non-simple) induced character for this case is as follows:

\begin{tabular}{c|ccccc|} 
Class & {$\left[1^{4}\right]$} & {$\left[1^{2} 2\right]$} & {$\left[2^{2}\right]$} & {$[13]$} & {$[4]$} \\
No. in Class & 1 & 6 & 3 & 8 & 6
\end{tabular}.

3. The induced trace matrix.

Let the traces or characters of the infinite sequence of induced representations $A^{[0]}, A^{[1]}, A^{[2]}, \ldots$ be taken as the rows of a rowfinite, column-infinite matrix $T$, the columns corresponding to cycle type. We shall call $T$ the induced trace-matrix.

For example by applying the rule of the last section we find the earlier rows of $T$, for $n=4$, to be as follows:

\begin{tabular}{|c|c|c|c|c|c|}
\hline Class & {$\left[1^{4}\right]$} & {$\left[\begin{array}{ll}1^{2} & 2\end{array}\right]$} & {$\left[2^{2}\right]$} & [13] & [4] \\
\hline No. in Class & 1 & 6 & 3 & 8 & 6 \\
\hline \multirow{7}{*}{$T=$} & 1 & 1 & 1 & 1 & 1 \\
\hline & 4 & 2 & • & 1 & $\cdot$ \\
\hline & 10 & 4 & 2 & 1 & . \\
\hline & 20 & 6 & * & 2 & . \\
\hline & 35 & 9 & 3 & 2 & 1 \\
\hline & 56 & 12 & - & 2 & $\cdot$ \\
\hline & 84 & 16 & 4 & 3 & - \\
\hline
\end{tabular}

Further continuation of the matrix $T$ suggests, what is actually the case, that the elements in the respective columns of $T$ are the coefficients of $1, x, x^{2}, \ldots$ in the expansions of

$(1-x)^{-4},(1-x)^{-2}\left(1-x^{2}\right)^{-1},\left(1-x^{2}\right)^{-2},(1-x)^{-1}\left(1-x^{3}\right)^{-1},\left(1-x^{4}\right)^{-1}$.

TheOREM 1. If $A_{i}$ is of cycle-type

$$
\left[p_{1}^{\alpha_{1}} p_{2}^{\alpha_{2}} \ldots\right] \text {, where } \Sigma p_{k} \alpha_{k}=n,
$$

then the traces of $A_{i}^{[r]}, r=0,1,2, \ldots$, are the coefficients of $1, x, x^{2}, \ldots$ in the expansion of

$$
\left\{\left(1-x^{p_{1}}\right)^{a_{1}}\left(1-x^{p_{2}}\right)^{a_{2}} \ldots\right\}^{-1}
$$


Proof. The characteristic determinant of $A_{i}$ is

$$
\left(1-x^{p_{1}}\right)^{a_{1}}\left(1-x^{\left.p_{2}\right)^{a_{2}}} \ldots \ldots\right.
$$

Now it is known (Franklin, 1894; Hurwitz, 1894') that the latent roots of $A^{[r]}$ are the $r$-ary powers and products of the latent roots of $A$. Hence the trace of $A^{[r]}$ is the sum of these, namely $h_{r}$, the complete homogeneous symmetric function of degree $r$ in the roots of $A$. But the generating function of the $h_{r}$ is the reciprocal of (1); and the theorem follows.

\section{Relation to the table of Simple characters.}

We consider the table $G$ of simple characters of the symmetric group on four symbols.

\begin{tabular}{|c|c|c|c|c|c|c|}
\hline \multirow{2}{*}{\multicolumn{2}{|c|}{$\begin{array}{c}\text { Class } \\
\text { No. in Class }\end{array}$}} & {$\left[1^{+}\right]$} & {$\left[1^{22}\right]$} & {$\left[2^{2}\right]$} & {$[13]$. } & [4] \\
\hline & & 1 & 6 & 3 & 8 & 6 \\
\hline \multirow{5}{*}{$G$} & {$[4]$} & 1 & 1 & 1 & 1 & 1 \\
\hline & [13] & 3 & 1 & -1 & & 1 \\
\hline & {$\left[2^{2}\right]$} & 2 & & 2 & -1 & . \\
\hline & {$\left[1^{2} 2\right]$} & 3 & -1 & -1 & . & 1 \\
\hline & {$\left[\mathbf{1}^{+}\right]$} & 1 & -1 & 1 & 1 & -1 \\
\hline
\end{tabular}

The rows give the simple characters, namely the traces of matrices belonging to the five irreducible representations typified by the partitions on the left. As we have seen, the rows of $T$, except for the first, are non-simple characters. Hence there is a premultiplying matrix $M$ (row-finite, column-infinite) such that $M G=T$.

Any particular element of $M$ (the rows and columns of which we suppose numbered $0,1,2 \ldots$ ) can be determined readily by means of the fundamental orthogonal relations, due to Frobenius, which exist between simple characters. For the symmetric group these may be expressed compactly by the statement

$$
G D G^{\prime}=n ! I,
$$

where $D$ is a diagonal matrix having in the diagonal the respective numbers of elements in the similar cycle-classes. Thus when $n=4$

$$
D=\operatorname{diag}[1,6,3,8,6] \text {. }
$$

\footnotetext{
1 References to results mentioned in the test are collected at the end of the paper.
} 
For example when $n=4$ the element $m_{43}$ in $M$ is found thus:

$(3 \overline{0} .1 .3-9.6 .1-3.3 .1+2.8 .0+1.6 .1) \div 4 !=2$, and in the same way the first several rows of $M$ are found to be:

\begin{tabular}{|c|c|c|c|c|c|}
\hline Reprn & [4] & [13] & {$\left[2^{2}\right]$} & {$\left[1^{2} 2\right]$} & {$\left[1^{4}\right]$} \\
\hline \multirow{7}{*}{$M=$} & 1 & & . & - & \\
\hline & 1 & 1 & & . & . \\
\hline & 2 & 2 & 1 & . & . \\
\hline & 3 & 4 & 1 & 1 & . \\
\hline & 5 & 6 & 3 & 2 & . \\
\hline & 6 & 10 & 4 & 4 & . \\
\hline & 9 & 14 & 7 & 6 & 1 \\
\hline
\end{tabular}

Further continuation of $M$ suggests that the elements in the respective columns are the coefficients of $1, x, x^{2}, \ldots$ in the expansions of

$$
\begin{gathered}
\left\{(1-x)\left(1-x^{2}\right)\left(1-x^{3}\right)\left(1-x^{4}\right)\right\}^{-1}, \quad x\left\{(1-x)^{2}\left(1-x^{2}\right)\left(1-x^{4}\right)\right\}^{-1}, \\
x^{2}\left\{(1-x)\left(1-x^{2}\right)^{2}\left(1-x^{4}\right)\right\}^{-1}, x^{3}\left\{(1-x)^{2}\left(1-x^{2}\right)\left(1-x^{4}\right)\right\}^{-1}, \\
x^{6}\left\{(1-x)\left(1-x^{2}\right)\left(1-x^{3}\right)\left(1-x^{4}\right)\right\}^{-1}
\end{gathered}
$$

These functions will be identified in the general case as being (except for a common polynomial factor) the "perpetuant" generating functions arrived at by $A$. Young in quantitative substitutional analysis.

\section{Bialternants and group characters.}

We begin from the fundamental result of Frobenius, by which an element $g_{p q}$ in the matrix $G$ of simple group characters is explicitly determined. Classes of similar cycle type are associated as usual with partitions

$$
[\pi]=\left[p_{1}^{a_{1}} p_{2}^{a_{2}} \ldots \ldots\right]
$$

irreducible representations with $n$-part partitions of $n$,

$$
[\lambda]=\left[\lambda_{1} \lambda_{2} \ldots \lambda_{n}\right] \text {. }
$$

Some of the earlier $\lambda$ 's will usually be zero. If rows (numbered $p=0,1,2, \ldots)$ correspond to the irreducible representations, and columns (numbercd $q=0,1,2, \ldots$ ) to the distinct classes, then $g_{p q}$ is the coefficient of

$$
x_{1}^{\lambda_{1}} x_{2}^{\lambda_{2}+1} \ldots x_{n^{n}}^{\lambda_{n}+n-1} \text { in } s_{p_{1}}^{a_{1}} s_{p_{2}}^{a_{2}} \ldots \Delta\left(x_{1}, x_{2}, \ldots, x_{n}\right),
$$


where $s_{r}$ is the ordinary power-sum symmetric function of the $x_{i}$, and $\Delta\left(x_{1}, x_{2}, \ldots, x_{n}\right)$ is the difference-product. Hence $g_{p q}$ must also be the coefficient of the alternating function

$$
\Sigma \pm x_{1}^{\lambda_{1}} x_{2}^{\lambda_{2}+1} \ldots x_{n^{n}}^{\lambda_{1}+n-1} \text { in } s_{p_{1}}^{\alpha_{1}} s_{p_{2}}^{a_{2}} \ldots \Delta\left(x_{1}, x_{2}, \ldots, x_{n}\right),
$$

where the summation is over all signed permutations of the $x_{i}$.

This classical result admits of more convenient statement; for it implies that $g_{p q}$ is the coefficient of

$$
\frac{A\left(\lambda_{1}, \lambda_{2}+1, \ldots, \lambda_{n}+n-1\right)}{A(0,1,2, \ldots, n-1)} \text { in } s_{p_{1}}^{a_{1}} s_{p_{2}}^{a_{2}} \ldots,
$$

where $A(\lambda, \mu, \nu, \ldots)$ denotes an alternant, and the right-hand side is expressed linearly by means of terms like the left-hand side. But such a quotient of alternants gives Jacobi's symmetric function, e.g.

$$
\frac{A\left(\begin{array}{lll}
0 & 3 & 4
\end{array}\right)}{A\left(\begin{array}{lll}
0 & 1 & 2
\end{array}\right)}=\left|\begin{array}{lll}
h_{0} & h_{3} & h_{4} \\
\cdot & h_{2} & h_{3} \\
\cdot & h_{1} & h_{2}
\end{array}\right|
$$

a "bialternant," expressible as an isobaric determinant with elements $h_{r}$, in the form

$$
\left|h_{\lambda_{i}-i+j}\right| \equiv h_{[\lambda]}
$$

where $[\lambda]$ indicates the partition of $n$ given by the diagonal suffixes. Hence, denoting isobaric products of power-sums by $s_{[\pi]}$, where [ $\left.\pi\right]$ is the partition of $n$ given by the suffixes of the factors $s_{r}$, we have the matrix relation

$$
G^{\prime}\left\{h_{[\lambda]}\right\}=\left\{s_{[\pi]}\right\} .
$$

Now any polynomial relation homogeneous, symmetric, and of degree $n$ in $n$ variables is true for any further number of variables. Let symmetric functions (distinguished by roman type) be constructed from the special infinite set of variables, the powers $1, x, x^{2}, \ldots$, where $x$ is of less than unit modulus, but otherwise arbitrary. For these we have evidently $s_{r}=\left(1-x^{r}\right)^{-1}$.

It follows, from Theorem 1 of $\S 3$, that if we postmultiply the row-infinite vector

$$
\left[x^{j}\right]=\left[\begin{array}{llll}
1 & x & x^{2} & \ldots
\end{array}\right]
$$

by the trace-matrix $T$ we obtain the row-finite vector [ $\left.\mathrm{s}_{[\pi]}\right]$, the elements of which are the isobaric power-sum products in (8) above. Again, transposing the relation $M G=T$ and postmultiplying it by the column vector $\left\{x^{j}\right\}$, we have

$$
G^{\prime} M^{\prime}\left\{x^{j}\right\}=T^{\prime}\left\{x^{j}\right\}=\left\{\mathrm{s}_{[\pi]}\right\} .
$$


Comparing now (10) with (8), we deduce

THEOREM 2. The elements in the respective columns of $M$ are the coefficients of $1, x, x^{2}, \ldots$ in the expansions of the bialternants $\mathrm{h}_{[\lambda]}$ in the variables $1, x, x^{2}, \ldots$

\section{Evaldation of the bialternants.}

It remains to find explicit expression for these bialternants. This has been by Young, who encountered them in substitutional analysis, and by D. E. Littlewood and A. R. Richardson in treating of special "S-functions" (bialternants). We shall essay here a rather different approach and order of derivation.

For the system of variables in question, the elementary symmetric functions $a_{r}$ are generated by the function of $t$,

$$
P(t ; x)=(1-t)(1-t x)\left(1-t x^{2}\right) \ldots,
$$

and the complete homogeneous symmetric functions $\mathrm{h}_{r}$ by the reciprocal of $P(t ; x)$. The latter is really a generating function of partitions, and we have the known relations ${ }^{1}$

$$
\begin{array}{r}
P(t ; x)=1-\frac{t}{1-x}+\frac{t^{2} x^{\frac{12}{2}(2-1)}}{(1-x)\left(1-x^{2}\right)}-\frac{t^{3} x^{\frac{1}{23}(3-1)}}{(1-x)\left(1-x^{2}\right)\left(1-x^{3}\right)}+\ldots, \\
\{P(t ; x)\}^{-1}=1+\frac{t}{1-x}+\frac{t^{2}}{(1-x)\left(1-x^{2}\right)}+\frac{t^{3}}{(1-x)\left(1-x^{2}\right)\left(1-x^{3}\right)}+\ldots
\end{array}
$$

whence

$$
\mathrm{a}_{r}=\frac{x^{\frac{1}{2} r(r-1)}}{(1-x)\left(1-x^{2}\right) \ldots\left(1-x^{r}\right)}, \quad \mathrm{h}_{r}=\frac{1}{(1-x)\left(1-x^{2}\right) \ldots\left(1-x^{r}\right)} .
$$

With an $n$-part partition of $n$, always in non-descending order of parts and so usually including zeros for the earlier parts,

$$
[\lambda]=\left[\lambda_{1} \lambda_{2} \ldots \lambda_{n}\right]
$$

we associate the set of numbers

$$
(\lambda+j)=\left(\begin{array}{llll}
\lambda_{1} & \lambda_{2}+1 \ldots \lambda_{n}+n-1
\end{array}\right)
$$

These numbers, which are in strictly ascending order, are the suffixes of elements in the first row of the bialternant $h_{[\lambda]}$; they are also the indices of the $x_{i}$ in Frobenius' generating function ( $\$ 5$ above) for the simple group characters. The dual set $(\lambda+j)^{\prime}$ with respect to an integer $m \geqq \lambda_{n}+n-1$ will be defined as the set obtained by taking those integers from 0 to $m$ which do not occur in $(\lambda+j)$,

1 MacMahon, Combinatory Analysis (1916), ii, 4, 10. 
subtracting them in turn from $m$, and arranging the results in ascending order. Usually we shall take $m=\lambda_{n}+n-1$. For example the dual of ( 236 ) with respect to 6 is (l 256$)$; with respect to 7 it is $(02367)$. Sets $(\lambda+j),(\lambda+j)^{\prime}$ corresponding to conjugate partitions are dual with respect to the same $m$.

With the partition $[\lambda]$ we associate also a certain index

$$
\sigma(\lambda)=(n-1) \lambda_{1}+(n-2) \lambda_{2}+\ldots+\lambda_{n-1} .
$$

The index for the conjugate partition

$$
[\lambda]^{\prime}=\left[1^{\lambda_{n}-\lambda_{n-1}} 2^{\lambda_{n-1}-\lambda_{n-2}} \ldots .\right]
$$

is readily found to be

$$
\sigma(\lambda)^{\prime}=\Sigma \frac{1}{2} \lambda(\lambda-1)
$$

THEOREM 3. If the variables are $1, x, x^{2}, \ldots$, then the bialternant

where

$$
\mathrm{h}_{[\lambda]}=x^{\sigma(\lambda)} \Delta\{\lambda+j\} / \Pi\{\lambda+j\} !,
$$

$$
\{a\} \equiv 1-x^{\alpha}, \quad\{a\} ! \equiv(1-x)\left(1-x^{2}\right) \ldots\left(1-x^{\alpha}\right),
$$

and $\Delta\{\lambda+j\}$ denotes the result of replacing typical factors $\beta-a, \beta>a$, in the ordinary difference product $\Delta(\lambda+j)$ by factors $1-x^{\beta-a}$.

Proof. Identities of the type

$$
\Delta\{\alpha \beta \gamma \delta\} \Delta\{\beta \gamma \delta \epsilon\} \Delta\{a \epsilon\}=\Delta\{\alpha \beta \gamma \delta \epsilon\} \Delta\{\beta \gamma \delta\}
$$

are verifiable for the general case by simple inspection.

From the expression for $h_{r}$ in (4) above, the theorem is true for bialternants of the first order. We assume it true for bialternants up to the order $p-1$. The extension to order $p$ may be illustrated without loss of generality by the case $p=4$. Using a fundamental identity in determinants, we have

$$
\begin{gathered}
h_{(\alpha \beta \gamma \delta)}=\left|\begin{array}{ll}
h_{(\alpha \beta \gamma)} & h_{(\beta \gamma \delta)} \\
h_{(\alpha-1, \beta-1, \gamma-1)} & h_{(\beta-1, \gamma-1, \delta-1)}
\end{array}\right| \div h_{(\beta-1, \gamma-1)} \\
=\left|\begin{array}{cc}
\frac{x^{2 a+(\beta-1)} \Delta\{a \beta \gamma\}}{\{a\} !\{\beta\} !\{\gamma\} !} & \frac{x^{2 \beta+(\gamma-1)} \Delta\{\beta \gamma \delta\}}{\{\beta\} !\{\gamma\} !\{\delta\} !} \\
\frac{x^{2(\alpha-1)+(\beta-2)} \Delta\{a \beta \gamma\}}{\{a-1\} !\{\beta-1\} !\{\gamma-1\} !} & \frac{x^{2(\beta-1)+(\gamma-2)} \Delta\{\beta \gamma \delta\}}{\{\beta-1\} !\{\gamma-1\} !\{\delta-1\} !}
\end{array}\right| \div \frac{x^{\beta-1} \Delta\{\beta \gamma\}}{\{\beta-1\} !\{\gamma-1\} !} \\
= \\
=x^{\sigma a+2(\beta-1)+(\gamma-2)} \Delta\{a \beta \gamma\} \Delta\{\beta \gamma \delta\} \Delta\{a \delta\} \\
\Delta\{\beta \gamma\}\{a\} !\{\beta\} !\{\gamma\} !\{\delta\} !
\end{gathered}
$$


The validity of the theorem for any order is thus established step by step.

As an example, consider the elements of the illustrative matrix $M$ of $\S 4$, in the column corresponding to [13]. The bialternant is

$$
\begin{aligned}
\mathrm{h}_{[13]}=\left|\begin{array}{ll}
\mathrm{h}_{1} \mathrm{~h}_{4} \\
\mathrm{~h}_{0} \mathrm{~h}_{3}
\end{array}\right| & =\frac{x\left(1-x^{3}\right)}{(1-x) \cdot(1-x)\left(1-x^{2}\right)\left(1-x^{3}\right)\left(1-x^{4}\right)} \\
& =x\left\{(1-x)^{2}\left(1-x^{2}\right)\left(1-x^{4}\right)\right\}^{-1} .
\end{aligned}
$$

The expansion of this function generates the elements in question.

In this same illustrative matrix $M$ the columns corresponding to conjugate partitions may be observed to contain the same non-zero elements in the same succession; they differ however in the number of zero elements which appear first of all. This depends on. a theorem concerning the conjugate bialternants $h_{[\lambda]}$ and $h_{[\lambda]^{\prime}}$ or $a_{[\lambda]}$. The number of zero elements at the heads of the respective columns will prove to be given by the conjugate indices $\sigma(\lambda)$ and $\sigma(\lambda)^{\prime}$.

$$
\text { ThEOREM 4. } \quad a_{\lambda]}=x^{\sigma(\lambda)^{\prime}} \Delta\{\lambda+j\} / \Pi\{\lambda+j\} ! \text {. }
$$

Proof. From the expression for $a_{r}$ in (4) above, the theorem is true for bialternants of the first order. We assume it is true up to order $p-1$, and proceed as in the previous theorem. We have

$$
\begin{aligned}
& a_{(a \beta \gamma \delta)}=\left|\begin{array}{ll}
a_{(a \beta \gamma)} & a_{(\alpha \beta \gamma)} \\
a_{(a-1, \beta-1, \gamma-1)} & a_{(\beta-1, \gamma-1, \delta-1)}
\end{array}\right| \div a_{(\beta-1, \gamma-1)} \\
& \frac{x^{\frac{1}{2[a(a-1)+(\beta-1)(\beta-2)+(\gamma-2)(\gamma-3)]} \Delta\{\alpha \beta \gamma\}}}{\{a\} !\{\beta\} !\{\gamma\} !} \quad \frac{x^{2[\beta(\beta-1)+(\gamma-1)(\gamma-2)+(\delta-2)(\delta-3)]} \Delta\{\beta \gamma \delta\}}{\{\beta\} !\{\gamma\} !\{\delta\} !} \\
& =\left|\frac{x^{[[(a-1)(a-2)+(\beta-2)(\beta-3)+(\gamma-3)(\gamma-4)]} \Delta\{a \beta \gamma\}}{\{a-1\} !\{\beta-1\} !\{\gamma-1\} !} \quad \frac{x^{3[(\beta-1)(\beta-2)+(\gamma-2)(\gamma-3)+(\delta-3)(\delta-4)]} \Delta\{\beta \gamma \delta\}}{\{\beta-1\} !\{\gamma-1\} !\{\delta-1\} !}\right| \\
& \div \frac{x^{2[1(\beta-1)(\beta-2)+(\gamma-2)(\gamma-3)]} \Delta\{\beta \gamma\}}{\{\beta-1\} !\{\gamma-1\} !} \\
& =\frac{x^{\frac{1}{2(\alpha-1)(a-2)+(\beta-2)(\beta-3)+(\gamma-3)(\gamma-4)+(\delta-3)(\delta-4)]} \Delta\{\alpha \beta \gamma\} \Delta\{\beta \gamma \delta\}}}{\Delta\{\beta \gamma\}\{\alpha\} !\{\beta\} !\{\delta\} !\{\delta\} !}\left|\begin{array}{lc}
x^{a-1+\beta-2+\gamma-3} x^{\beta-1+\gamma-2+\delta-3} \\
1-x^{\alpha} & 1-x^{\delta}
\end{array}\right|
\end{aligned}
$$

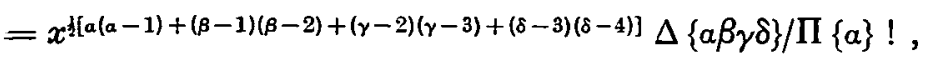

$$
\begin{aligned}
& \text { by }(10) \text {. }
\end{aligned}
$$

The validity of the theorem for any order is thus established as before. 
An immediate consequence of Theorems 3 and 4 is

THEOREM 5 .

$\Delta\{\lambda+j\} / \Pi\{\lambda+j\} !$

is invariant for dual sets (conjugate partitions).

This is a theorem of Young, and is proved directly by him. It corresponds to the theorem (in ordinary difference products and ordinary factorials) that the function

$$
\Delta(\lambda+j) / \Pi(\lambda+j) !
$$

is invariant for dual sets; this function being the enumerant of Young's standard tableaux for the partitions in question or, equally well, the order of the corresponding irreducible matrix representations of the symmetric group of order $n$ !, in fact the $f_{(\lambda)}$ of Frobenius.

Returning to the matrix $M$, let the index $\sigma(\lambda)$ be determined for the various partitions of $n$. The rows of $M$ which are ordinally numbered by these values $\sigma(\lambda)$ will contain $1,2,3, \ldots, P(n)$ nonzero elements respectively, where $P(n)$ is the number of partitions of $n$. They are therefore linearly independent. Picking these rows out, we may construct from them a non-singular matrix $R$ of triangular shape in its non-zero elements; and constructing a matrix $U$ in the same way from the corresponding rows of the trace-matrix $T$, we have the relation

$$
R G=U
$$

whence, from the triangular nature of $R$, the table $G$ of simple characters may easily be found, row by row.

Thus in the case $n=4$ the relation $R G=U$ appears as

$$
\left[\begin{array}{rrrrr}
1 & . & . & \cdot & \cdot \\
1 & 1 & . & \cdot & \cdot \\
2 & 2 & 1 & . & \cdot \\
3 & 4 & 1 & 1 & \cdot \\
9 & 14 & 7 & 6 & \cdot
\end{array}\left[\begin{array}{rrrrr}
1 & 1 & 1 & 1 & 1 \\
3 & 1 & -1 & . & -1 \\
2 & . & 2 & -1 & \cdot \\
3 & -1 & -1 & . & 1 \\
1 & -1 & 1 & 1 & -1
\end{array}\right]=\left[\begin{array}{rrrrr}
1 & 1 & 1 & 1 & 1 \\
4 & 2 & . & 1 & . \\
10 & 4 & 2 & 1 & . \\
20 & 6 & . & 2 & . \\
84 & 16 & 4 & 3 & . \\
- & & & &
\end{array}\right] .\right.
$$

\section{Compodnd PERMUTATion matrices.}

The study of the trace-matrix of compound matrices is not 60 interesting. If we include the extreme compounds $A^{(0)}=1, A^{(n)}=|A|$, the number of compound matrices of any matrix $A$ of order $n$ is only 
$n+1$, whereas the number of irreducible representations of the symmetric group of order $n !$ is $P(n)$; and for $n>4, P(n)$ exceeds $n+1$. Hence the information concerning the symmetric group yielded by the trace-matrix of compound permutation matrices is in general inadequate. It has been shown, however, by D. E. Littlewood, and A. R. Richardson, that the trace-matrices of compound permanents and immanants (determinantal functions bearing to determinants and permanents the same relation that bialternants bear to symmetric functions $a_{r}$ and $h_{r}$ ) remedy this deficiency.

The trace-matrix $T$ for compounds of permutation matrices is easily found. Since the latent roots of $A^{(r)}$ are the $r$-ary products without repetitions (theorem of $\operatorname{Rados}, 1891$ ) of the latent roots of $A$, the trace of $A^{(r)}$ is the elementary symmetric function of degree $r$ in those roots, namely $a_{r}$. But the generating function of the $a_{r}$ is the characteristic determinant of $A$. As has been seen in $\S 1$, the characteristic determinants for permutation matrices $A_{i}$ are of the form

$$
\left(1-x^{p_{1}}\right)^{a_{1}}\left(1-x^{p_{2}}\right)^{\alpha_{2}} \ldots
$$

It follows at once that the coefficients of $1, x, x^{2}, \ldots$ in the expansions of these multi-binomials for the respective cycle-types are the elements in the corresponding columns of the trace-matrix $T$ for compounds.

The nature of the premultiplying matrix $M$ in the relation $M G=T$ is easy to determine, but without significance, since for $n>4$ the rank of $M$ is less than $P(n)$.

A more general question of some interest is what relation the "Invariant matrices" (in the sense of Schur's classic dissertation) of permutation matrices bear to the simple characters of the symmetric group. We reserve this for further consideration.

\section{REFERENCES.}

Franklin, F. Amer. Jour. Math., 16 (1894) 205-6.

Frobenius, G. Berlin Sitzungsb., (1900) 518-9.

Hurwitz, A. Math. Ann. 45 (1894) 381-404 (391).

Littlewood, D. E. and Richardson, A. R. Quart. J. of Math. (Oxford Series) 6 (1935) $185,193$.

Littlewood, D. E. and Richardson, A. R. Phil. Trans. Roy. Soc. (A) 233 (1934) 99-141 (101-113). 
MacMahon, P. A. Combinatory Analysis (1916), Vol. ii, Chapter 1.

Muir, T. History of Determinants, Vol. 1, 341.

Schur, I. Berlin Dissertation (1901), Ueber eine Klasse ron Matrizen, 49-52 passim.

Young, A. Phil. Trans. Roy. Soc. (A) 234 (1935) 79.114 (80.86).

[Added 14th January, 1936. It has been kindly brought to my notice by a referee that the method given in this paper $(\$ 6)$ for finding simple characters is not entirely independent of that given by D. E. Littlewood and A. R. Richardson, Quart. J. of Math. (Oxford Series) 6 (1935), 192, the case of Theorem VII as there enunciated being taken for which $N \rightarrow \infty$. I wish therefore to draw attention to this fact, while remarking that the evaluation of simple characters was a secondary issue, the chief interest being in the properties of a class of invariant matrices.-A. C. A.] 